OPEN ACCESS

Edited by:

Heike Wulff,

University of California, Davis,

United States

Reviewed by:

Sunil Jit Ramamoorthy Jeewanlal Logantha,

University of Liverpool,

United Kingdom

Benoit Tyl,

Institut de Recherche International

Servier, France

*Correspondence:

Alina Scridon

alinascridon@gmail.com

Specialty section:

This article was submitted to Cardiovascular and Smooth

Muscle Pharmacology,

a section of the journal

Frontiers in Pharmacology

Received: 20 August 2020

Accepted: 26 February 2021

Published: 08 April 2021

Citation:

Scridon A, Halaţiu VB, Balan Al, Cozac DA, Moldovan V, Bănescu C, Perian M and Şerban RC (2021) LongTerm Effects of Ivabradine on Cardiac Vagal Parasympathetic Function in Normal Rats.

Front. Pharmacol. 12:596956. doi: 10.3389/fphar.2021.596956

\section{Long-Term Effects of Ivabradine on Cardiac Vagal Parasympathetic Function in Normal Rats}

\author{
Alina Scridon ${ }^{1,2 *}$, Vasile Bogdan Halaţiu ${ }^{1}$, Alkora loana Balan ${ }^{1}$, Dan Alexandru Cozac ${ }^{1,3}$, \\ Valeriu Moldovan ${ }^{1,2}$, Claudia Bănescu ${ }^{1,2}$, Marcel Perian ${ }^{1,2}$ and Răzvan Constantin Şerban ${ }^{3}$ \\ 'University of Medicine, Pharmacy, Science and Technology "George Emil Palade" of Târgu Mureș, Târgu Mureș, Romania, \\ ${ }^{2}$ Center for Advanced Medical and Pharmaceutical Research, Târgu Mureș, Romania, ${ }^{3}$ Emergency Institute for Cardiovascular \\ Diseases and Transplantation Târgu Mureş, Târgu Mureş, Romania
}

Background: The complex interactions that exist between the pacemaker current, $I_{\mathrm{f}}$, and the parasympathetic nervous system could significantly influence the course of patients undergoing chronic therapy with the $I_{\mathrm{f}}$ blocker ivabradine. We thus aimed to assess the effects of chronic ivabradine therapy on autonomic modulation and on the cardiovascular response to in situ and in vitro parasympathetic stimulation. The right atrial expression of HCN genes, encoding proteins for $I_{\mathrm{f}}$, was also evaluated.

Methods: Sympathetic and parasympathetic heart rate variability parameters and right atrial $H C N(1-4)$ RNA levels were analyzed in 6 Control and 10 ivabradine-treated male Wistar rats (IVA; 3 weeks, $10 \mathrm{mg} / \mathrm{kg} /$ day). The heart rate (HR) and systolic blood pressure (SBP) responses to in situ electrical stimulation of the vagus nerve $(2-20 \mathrm{~Hz})$ were assessed in 6 additional Control and 10 IVA rats. The spontaneous sinus node discharge rate (SNDR) response to in vitro cholinergic receptors stimulation using carbamylcholine $\left(10^{-9}-10^{-6} \mathrm{~mol} / \mathrm{L}\right)$ was also assessed in these later rats.

Results: Ivabradine significantly increased vagal modulation and shifted the sympathovagal balance toward vagal dominance. In Control, in situ vagus nerve stimulation induced progressive decrease in both the SBP $(p=0.0001)$ and the HR $(p<0.0001)$. Meanwhile, in IVA, vagal stimulation had no effect on the $\mathrm{HR}(p=0.16)$ and induced a significantly lower drop in SBP ( $p<0.05)$. IVA also displayed a significantly lower SNDR drop in response to carbamylcholine $(p<0.01)$ and significantly higher right atrial HCN4 expression $(p=0.02)$.

Conclusion: Chronic ivabradine administration enhanced vagal modulation in healthy rats. In addition, ivabradine reduced the HR response to direct muscarinic receptors stimulation, canceled the cardioinhibitory response and blunted the hemodynamic response to in situ vagal stimulation. These data bring new insights into the

\footnotetext{
Abbreviations: ANS, autonomic nervous system; GAPDH, glyceraldehyde 3-phosphate dehydrogenase; HCN, hyperpolarization-activated cyclic nucleotide-gated channels; HF, high-frequency; HR, heart rate; HRV, heart rate variability; LF, lowfrequency; NS, non-significant; pNN5, percentage of successive RR intervals that differ by $>5$ ms; RMSSD, root mean square of the successive RR-interval differences; SBP, systolic blood pressure; SDNN, standard deviation of normal RR intervals; SNDR, sinus node discharge rate.
} 
mechanisms of ivabradine-related atrial proarrhythmia and suggest that long-term $I_{\mathrm{f}}$ blockade may protect against excessive bradycardia induced by acute vagal activation.

Keywords: cardioinhibitory response, $\mathrm{HCN} 4, I_{\mathrm{f}}$ current, ivabradine, vagal tone

\section{INTRODUCTION}

The heart rate is the result of a highly coordinated sequence of electrical phenomena that normally take place in the pacemaker cells of the sinus node. The autonomic nervous system (ANS) ensures both long-term, tonic control and short-term, reflex adaptation of the HR to internal and external factors. The hyperpolarization-activated inward current $\left(I_{\mathrm{f}}\right)$ is critical in establishing the HR and one of the most relevant targets of HR modulation by the ANS (DiFrancesco, 2010).

Ivabradine is a specific $I_{\mathrm{f}}$ blocker devoid of dromotropic, inotropic, and lusitropic effects (Camm and Lau, 2003; Manz et al., 2003). In patients with heart failure, chronic ivabradine administration significantly improved clinical outcomes (Swedberg et al., 2010), while also causing a significant, although modest increase in atrial fibrillation, but not in ventricular arrhythmias occurrence (Martin et al., 2014; Cammarano et al., 2016). Studies have also shown that, by inhibiting $I_{\mathrm{f}}$, ivabradine can significantly reduce the tachycardic response to acute sympathetic stimulation and can thus provide benefit in a wide range of settings associated with sympathetic hyperactivation. In patients with postural orthostatic tachycardia syndrome, ivabradine significantly improved the quality of life (Taub et al., 2021) and efficiently reduced the HR at rest and during tilting (Gee et al., 2018; Taub et al., 2021). In a small randomized controlled trial, ivabradine therapy significantly improved symptoms in patients with inappropriate sinus tachycardia (Cappato et al., 2012). Promising results have also been reported in patients with sinus tachycardia-mediated vasovagal syncope, in whom ivabradine was well tolerated and was associated with marked benefit or complete resolution of symptoms (Sutton et al., 2014).

The relationship between $I_{\mathrm{f}}$ blockade and parasympathetic activation appears to be, however, much more complex. In rats with post-myocardial infarction heart failure, long-term ivabradine therapy was shown to counteract the increase in the expression of genes encoding for hyperpolarizationactivated cyclic nucleotide-gated (HCN) channels, responsible for generating $I_{\mathrm{f}}$, in the ventricular myocardium (Suffredini et al., 2012). Meanwhile, chronic ivabradine administration was associated with a significant increase in sinus node $\mathrm{HCN} 4$ expression in mice (Leoni et al., 2006) If ivabradine exerts such an effect on sinus node cells, this could significantly alter the HR response to vagal stimulation. Acute (Mangin et al., 1998), but not chronic (Silva et al., 2016) intraperitoneal ivabradine administration has also been associated with marked increase in heart rate variability (HRV) parameters in rats. In addition, clinical and experimental data indicate that in the setting of heart failure, long-term ivabradine therapy shifts the sympathovagal balance toward vagal dominance (Milliez et al., 2009; Kurtoglu et al., 2014; Böhm et al., 2015). Accumulating data therefore suggest that chronic ivabradine therapy could alter both the vagal modulation and the HR response to vagal stimulation and could thus influence the risk of patients prone to cardiac arrhythmias and the clinical course of patients with vaso-vagal syncope. However, the effects of chronic $I_{\mathrm{f}}$ blockade on the HR response to acute parasympathetic stimulation have not been evaluated to date and its impact on vagal modulation in settings other than heart failure remains to date unknown.

We therefore aimed to help solving some of the existing controversies and to increase knowledge regarding the effects of the $I_{\mathrm{f}}$ blocker ivabradine. To achieve this goal, we designed an experimental study to assess the effects of chronic ivabradine therapy on the $\mathrm{HR}$ response to in situ and in vitro acute parasympathetic stimulation. The impact of chronic ivabradine administration on the sympatho-vagal modulation and on the right atrial expression level of $\mathrm{HCN}$ channels was also evaluated.

\section{MATERIALS AND METHODS}

\section{Studied Animals}

The flow chart of the study design is presented in Figure 1. Adult male Wistar rats (200-250 g) obtained from the local animal facility were initially randomized into two groups: Control $(n=12)$ and IVA $(n=$ 20). All animals were housed individually in polycarbonate cages, in a temperature-controlled room $\left(21-24^{\circ} \mathrm{C}\right)$, with a $12 / 12 \mathrm{~h}$ light/dark cycle, and had free access to water and standard food. Rats in the IVA group received a daily dose of $10 \mathrm{mg} / \mathrm{kg}$ of body weight of ivabradine (ivabradine hydrochloride; Servier; Courbevoie, France) in their drinking water throughout the study, starting three weeks prior to any experimental procedure. To ensure that each rat consumed the adequate amount of ivabradine, the drug was dissolved in $15 \mathrm{ml}$ of water; as soon as this amount was consumed, normal tap water was put at the rats' disposal for the rest of the day. Control rats received normal tap water throughout the study. The daily water intake was measured in all rats throughout the study. All protocols complied with the International Council for Laboratory Animal Science guidelines (Directive 2010/63/EU) and were approved by the local Ethics Committee and the National Sanitary Veterinary and Food Safety Authority. Partial blinding was applied in the present study: the researchers who performed the experimental procedures, (i.e., ECG device implantation, vagus nerve stimulation, atrial sampling, in vitro studies) were not blinded to the study group, but all parameters were measured and all statistical analyses were performed in a blinded manner.

\section{Continuous $24 \mathrm{~h}$ ECG Monitoring and Heart Rate Variability Analysis}

Three weeks after the beginning of the study, Control and IVA rats were re-randomized into two subgroups each. Six Control 


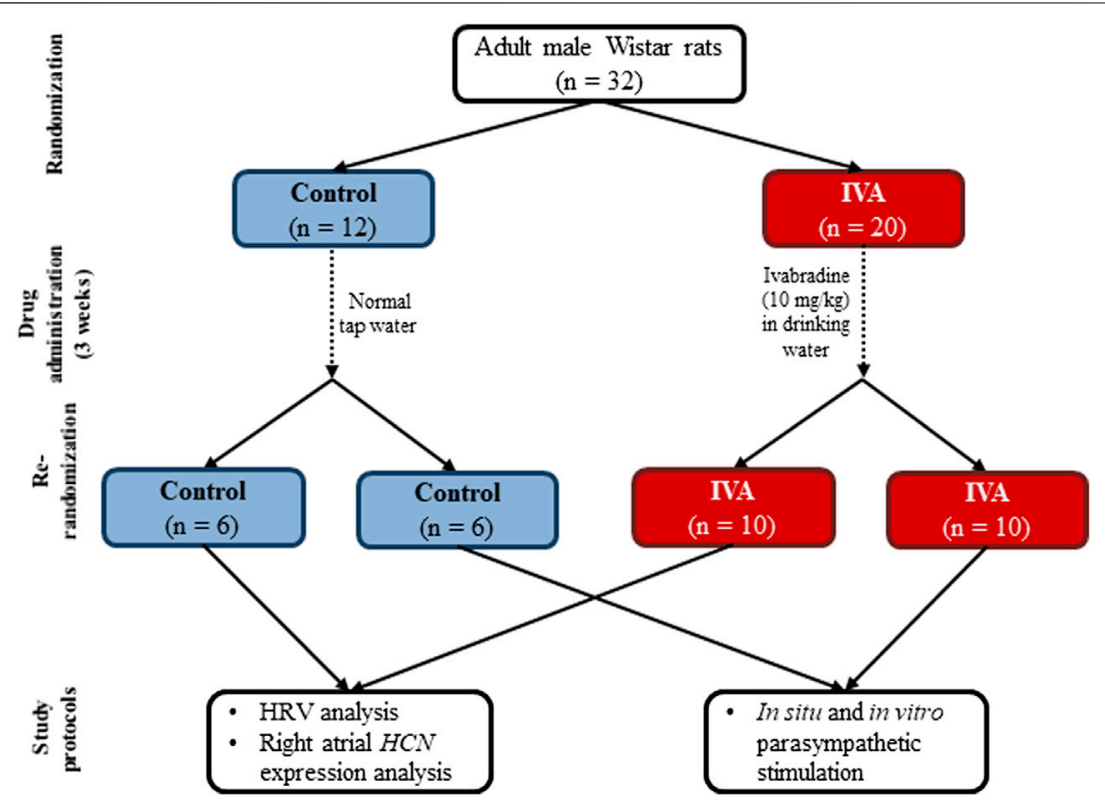

FIGURE 1 | The flow chart of the study design for the Control and the ivabradine-treated (IVA) groups. HRV - heart rate variability

and 10 IVA rats were implanted with radiotelemetry ECG devices (TA11 CA-F40; Data Sciences International, St. Paul, MN), as described previously (Doñate Puertas et al., 2017). After one week of post-implantation recovery, $24 \mathrm{~h}$ continuous ECG monitoring was performed in unrestrained, "conscious" rats, and the mean baseline HR was calculated using a program developed to automatically detect the $\mathrm{R}$ waves and measure the RR intervals (Gallet et al., 2013). Heart rate variability analysis was performed based on the $24 \mathrm{~h}$ ECG recordings by analyzing beat-to-beat variations in $\mathrm{RR}$ intervals in the time and frequency domains, as described previously (Scridon et al., 2012). For the time domain analysis, the standard deviation of normal RR intervals (SDNN), the root mean square of the successive RR-interval differences (RMSSD), and the percentage of successive $\mathrm{RR}$ intervals that differed by $>$ $5 \mathrm{~ms}$ (pNN5) were assessed. For the frequency domain analysis, time series were resampled at $20 \mathrm{~Hz}$ using a cubic spline, then spectral power in the low-frequency (LF) band $(0.3-0.6 \mathrm{~Hz})$, the high-frequency $(\mathrm{HF})$ band $(0.6-2.5 \mathrm{~Hz})$, and the LF/HF ratio were estimated on 2,048-point (102.4 s) segments windowed by the Hanning function and overlapping by $50 \%$ using a fast Fourier transform. All ECG tracings were assessed visually and all artifacts, arrhythmic events, and compensatory pauses were excluded prior to HRV analysis.

\section{Right Atrial Expression Analysis of Genes Encoding for Hyperpolarization-Activated Cyclic Nucleotide-Gated Channels}

At the end of the ECG monitoring period, the 6 Control and 10 IVA rats were euthanized using an intraperitoneal injection of a terminal dose of sodium pentobarbital $(>100 \mathrm{mg} / \mathrm{kg})$. The thoracic cavity was opened and the heart was removed. The free wall of the right atrium was collected and rapidly immersed into RNA stabilization solution (RNAlater; Thermo Fisher Scientific, Waltham, MA). The RNA was isolated using iPrep PureLink Total RNA Kits and the iPrep Purification Instrument (Thermo Fisher Scientific). Reverse transcription was performed using the SuperScript VILO cDNA Synthesis Kit (Thermo Fisher Scientific). The RNA expression levels of three target genes, (i.e. HCN1, HCN2, and HCN4) and one control gene, (i.e. glyceraldehyde 3phosphate dehydrogenase $[G A P D H]$ ) were analyzed using a customized fast 96-well plate containing TaqMan Gene Expression Assays for the tested genes (Thermo Fisher Scientific). The expression of the neuron-specific HCN3 isoform was also analyzed. All experiments were performed on a 7500 Fast Dx Real-Time PCR System (Applied Biosystems, Waltham, MA). The expression levels of HCN1, HCN2, HCN3, and HCN4 were normalized with GAPDH housekeeping gene levels and compared between the two groups.

\section{In situ and in vitro Parasympathetic Stimulation}

Three weeks after the beginning of the study, the remaining 6 Control and 10 IVA rats were submitted to vagus nerve stimulation under ketamine/medetomidine anesthesia (i.p., $75.0 / 0.5 \mathrm{mg} / \mathrm{kg}$ ). Briefly, the anterior cervical region was dissected and the sternohyoid and sternocleidomastoid muscles were separated and retracted laterally to allow visualization of the carotid artery. The right vagus nerve was carefully isolated from 
the surrounding connective tissue. In order to avoid interferences from retrograde electrical vagus nerve stimulation, the nerve was secured using two surgical threads and was then cut. A stimulation electrode was placed beneath the distal end of the vagus nerve and was elevated to avoid contact with the surrounding tissues. The nerve was then stimulated electrically using rectangular impulses (pulse duration $0.5 \mathrm{~ms} ; 20 \mathrm{~V}$ ) at progressively higher frequencies, (i.e. 2, 5, 10, and $20 \mathrm{~Hz}$ ). Each stimulation protocol was applied for $15 \mathrm{~s}$, with $5 \mathrm{~min}$ intervals between stimulations. Surface ECG was continuously recorded during the entire duration of the protocol and the HR was calculated at baseline and during each stimulation protocol based on RR intervals duration. The ECG signal was captured using three electrodes placed on the two upper limbs and on the left lower limb, was amplified, and delivered to the acquisition board. The ECG signal was recorded using an acquisition program developed using the LabVIEW 8.20 software (National Instruments, Austin, TX). Systolic blood pressure (SBP) was measured non-invasively at baseline and during each stimulation protocol using a photoplethysmographic method, as described previously (Scridon et al., 2015). Briefly, a pneumatic tail cuff was placed proximally on the rat's tail and inflated/deflated using the PE-300 programmed electrosphygmomanometer (Narco Bio-Systems Inc., Houston, TX). The photoplestimography sensor was placed on the tail distally to the pneumatic cuff, with the infrared beam at the level of the caudal artery. The cuff pressure and the phototransducer signals were routed to the signal acquisition board. The signals were recorded using an acquisition program developed using the LabVIEW 8.20 software (National Instruments).

At the end of the stimulation protocols, the anesthetized rats were euthanized by thoracotomy. The hearts were explanted and rapidly immersed into prewarmed $\left(37^{\circ} \mathrm{C}\right)$ oxygenated $\left(95 \% \mathrm{O}_{2}\right.$; $\left.5 \% \quad \mathrm{CO}_{2}\right) \quad$ Krebs-Henseleit solution containing $\mathrm{NaCl}$ (118.00 mM), $\mathrm{KCl}(4.70 \mathrm{mM}), \mathrm{NaHCO}_{3}$ (25.00 mM), $\mathrm{MgSO}_{4}$ (1.20 mM), $\mathrm{CaCl}_{2}(1.25 \mathrm{mM}), \mathrm{KH}_{2} \mathrm{PO}_{4}(1.20 \mathrm{mM})$, and glucose $(11.00 \mathrm{mM})$. The right atrium was isolated and transferred into the Steiert organ bath (Hugo Sachs Elektronik-Harvard Apparatus; March-Hugstetten, Germany) containing oxygenated Krebs-Henseleit solution at $37^{\circ} \mathrm{C}$. The spontaneous sinus node discharge rate was measured at baseline and after direct cholinergic receptors stimulation using carbamylcholine solutions with progressively higher concentrations $\left(10^{-9} \mathrm{~mol} / \mathrm{L}\right.$ to $10^{-6} \mathrm{~mol} / \mathrm{L}$, prewarmed at $37^{\circ} \mathrm{C}$ ). Each solution was applied for $10 \mathrm{~min}$. All samples were washed for $5 \mathrm{~min}$ with oxygenated Krebs-Henseleit solution between exposures to the successive carbamylcholine solutions.

\section{Statistical Analysis}

Statistical analyses were undertaken using MedCalc for Windows, version 12.4.3.0 (MedCalc Software; Ostend, Belgium). A two-tailed $p$-value $<0.05$ was considered statistically significant. All data were tested for normality and are expressed as means \pm standard error of the mean or median and interquartile range, as appropriate. Betweengroup comparisons were performed using the unpaired Student's $t$-test or the Mann-Whitney $U$-test, as appropriate. Differences within the same group were tested for significance using the paired Student's $t$-test or the Wilcoxon matched-pairs signed-rank test, as appropriate, and repeated-measures ANOVA. Changes in the spontaneous sinus node discharge rate in response to carbamylcholine administration were also analyzed by a nonparametric two-way ANOVA, factoring for the effects of ivabradine treatment status (ivabradine-treated vs. nontreated) and carbamylcholine concentrations $\left(10^{-9} \mathrm{~mol} /\right.$ $\left.\mathrm{L}-10^{-6} \mathrm{~mol} / \mathrm{L}\right)$. Due to the very limited amount of data available on this topic, the sample size could not be calculated prior to the study. However, based on previous studies in non-treated rats, Control rats were expected to present low interindividual variability and a sample size of 6 was therefore considered sufficient for these groups. To compensate for potentially higher interindividual differences in the ivabradine-treated animals, the two IVA groups were designed larger $(n=10)$ than the Control groups.

\section{RESULTS}

There was no significant difference in the daily water intake between the IVA and the Control rats $(53.21 \pm 2.49 \mathrm{ml} / 24 \mathrm{~h}$ vs. $57.50 \pm 3.30 \mathrm{ml} / 24 \mathrm{~h} ; p=0.34)$.

\section{Chronic Ivabradine Administration Increases Vagal Modulation and Shifts the Sympatho-Vagal Balance Toward Vagal Dominance in Healthy Rats}

Figure 2 depicts typical telemetric ECG tracings recorded in Control and IVA rats. As expected, mean $24 \mathrm{~h}$ HR was significantly lower in the ivabradine-treated rats compared to their non-treated counterparts $(p<0.01$; Table 1). Mean awake and mean asleep HR were both significantly lower in the ivabradine-treated compared to the non-treated rats (both $p<$ 0.0001; Table 1). In the Control rats, there was a $22.6 \pm 2.8 \mathrm{bpm}$ $(p<0.001)$ awake/asleep HR difference, compared with an $18.7 \pm$ $5.9 \mathrm{bpm}(p=0.01)$ awake/asleep HR difference in the ivabradinetreated rats $(p=0.63)$.

Similarly to what was previously reported in the setting of heart failure (Milliez et al., 2009; Kurtoglu et al., 2014; Böhm et al., 2015), HRV analysis revealed a significant increase in vagal modulation and a shift of the sympatho-vagal balance toward vagal dominance in ivabradine-treated healthy rats (Table 1). The RMSSD, pNN5, and the HF components of the HRV spectrum, reflecting vagal modulation, were all significantly higher in the IVA compared with the Control rats (awake, asleep, and over $24 \mathrm{~h}$; all $p<0.05$; Table 1). The LF/HF ratio, an index of sympathetic and parasympathetic interactions, was significantly lower in the IVA than in the Control rats (awake, asleep, and over $24 \mathrm{~h}$; all $p<0.05$; Table 1), demonstrating a shift of the sympatho-vagal balance toward vagal dominance in the ivabradine-treated rats. No significant change was observed in the LF components of the HRV spectrum (awake, asleep, and over 24 h; all $p=$ NS; Table 1). 

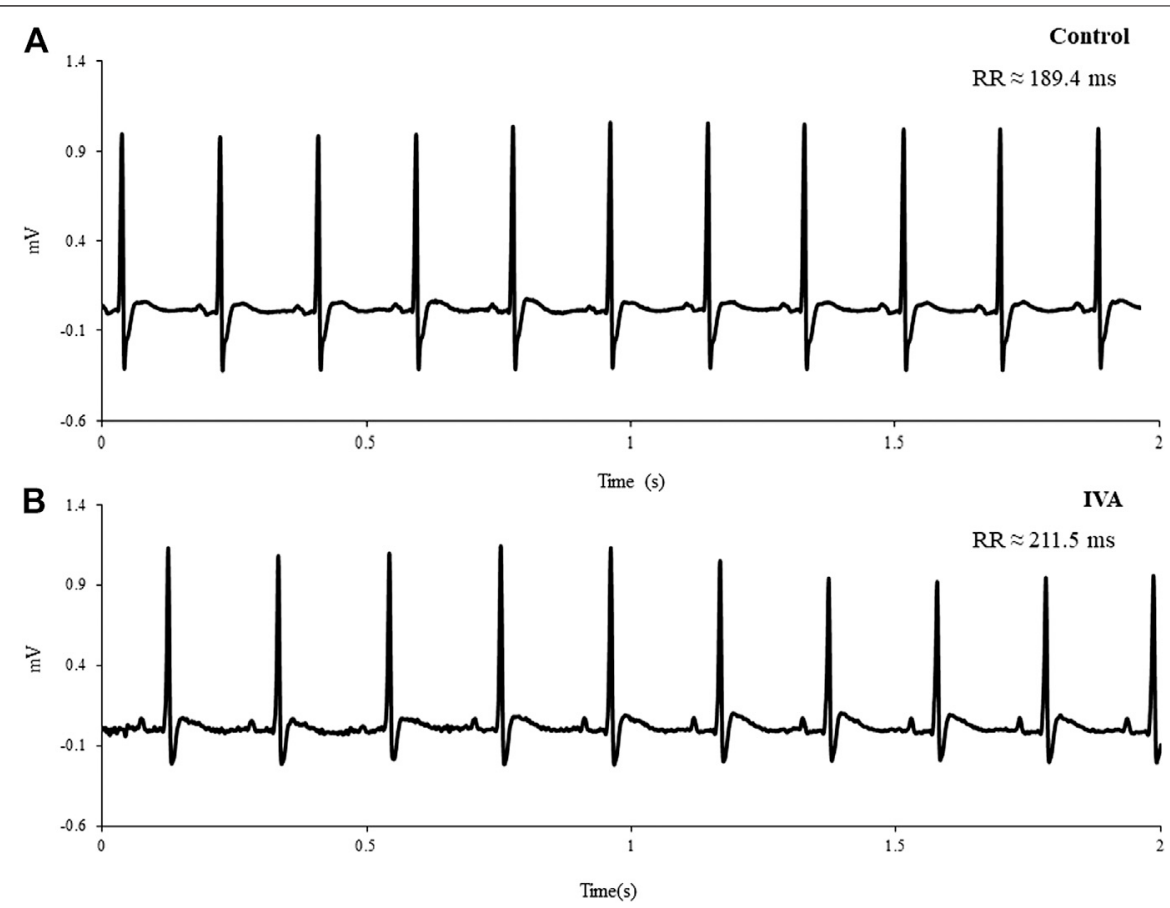

FIGURE 2 | Representative telemetric ECG tracings recorded in a Control (A) and an ivabradine-treated (IVA) rat (B)

\section{Chronic Ivabradine Administration} Up-Regulates the Right Atrial Expression of the Hyperpolarization-Activated Cyclic Nucleotide-Gated Channel 4

Right atrial HCN1 and HCN2 expression levels were similar (both $p=\mathrm{NS}$ ) between the IVA and the Control rats (Figure 3). There was also no significant difference in the right atrial expression of the neuron-specific HCN3 isoform between the two groups ( $p=$ 0.22; Figure 3). However, the right atrial expression of HCN4, the most highly expressed $\mathrm{HCN}$ isoform in the sinus node (Sartiani et al., 2017), was significantly higher in the ivabradine-treated compared to the non-treated rats $(p=0.02$; Figure 3).

\section{Long-term Ivabradine Administration Cancels the Heart Rate Response and Reduces the Blood Pressure Response to Acute in situ Vagal Stimulation}

As expected, baseline HR (derived from surface ECG recordings performed in anesthetized rats prior to vagus nerve sectioning) was significantly lower in the IVA compared with the Control rats (202.2 \pm 6.6 bpm vs. $246.3 \pm 13.6$ bpm; $p=0.01)$, whereas SBP was similar between the two groups $(143.5 \pm 6.9 \mathrm{mmHg}$ vs. $154.1 \pm$ $9.8 \mathrm{mmHg} ; p=0.58$ ).

In the Control rats, electrical stimulation of the vagus nerve induced a significant, progressive decrease in both the SBP ( $p=$ $0.0001)$ and the HR $(p<0.0001)$ (Figure 4). In the IVA rats, electrical stimulation of the vagus nerve also induced a significant, progressive decrease in the SBP $(p<0.0001)$ (Figure 5). However, in the ivabradine-treated rats, vagus nerve stimulation had no effect on the HR ( $p=0.16$; Figure 5). In addition, the decrease in SBP in response to vagus nerve stimulation was also significantly lower in the IVA compared with the Control rats for all four stages of the stimulation protocol (all $p<0.05$; Figure 6).

\section{Ivabradine Reduces the Drop in the Sinus Node Discharge Rate in Response to in vitro Cholinergic Receptors Stimulation}

When right atrial samples were assessed in vitro under progressively higher carbamylcholine concentrations, both IVA and Control rats displayed a significant, progressive decrease in the spontaneous discharge rate of the sinus node (both $p<0.0001$; Figure 7). There was no significant difference in the sinus node discharge rate drop in response to similar carbamylcholine concentrations between the two groups $(p=$ NS for all carbamylcholine concentrations; Figure 7). However, in absolute value, the drop in the sinus node discharge rate was $\sim 30-40 \mathrm{bpm}$ lower in the ivabradine-treated than in the nontreated rats for all carbamylcholine concentrations (Figure 7). Moreover, when the responses of the sinus node discharge rate to all carbamylcholine concentrations were combined, the decrease in the spontaneous discharge rate of the sinus node in response to carbamylcholine administration was significantly lower in the IVA compared with the Control rats $(p<0.01$; Figure 7$)$. In addition, two-way ANOVA factoring for the effects of ivabradine treatment status (ivabradine-treated vs. non-treated) and carbamylcholine concentrations $\left(10^{-9} \mathrm{~mol} / \mathrm{L}-10^{-6} \mathrm{~mol} / \mathrm{L}\right)$ demonstrated that the response of the spontaneous discharge 
TABLE 1 | Mean $24 \mathrm{~h}$ heart rate and heart rate variability parameters in ivabradinetreated (IVA) and non-treated (Control) rats.

\begin{tabular}{|c|c|c|c|}
\hline Parameter & Control $(n=6)$ & IVA $(n=10)$ & $p$-value \\
\hline \multicolumn{4}{|l|}{ Heart rate $(H R)$} \\
\hline Mean 24 h HR (bpm) & $341.5 \pm 8.3$ & $301.3 \pm 7.5$ & $<0.01$ \\
\hline Mean HR awake (bpm) & $356.6 \pm 5.5$ & $304.6 \pm 6.9$ & $<0.0001$ \\
\hline Mean HR asleep (bpm) & $334.0 \pm 6.4$ & $285.9 \pm 4.9$ & $<0.0001$ \\
\hline \multicolumn{4}{|c|}{24 h Heart rate variability analysis } \\
\hline SDNN (ms) & $23.3 \pm 1.9$ & $26.6 \pm 1.7$ & 0.23 \\
\hline RMSSD (ms) & $3.9 \pm 0.3$ & $5.5 \pm 0.2$ & $<0.01$ \\
\hline pNN5 (\%) & $16.9 \pm 2.6$ & $28.1 \pm 1.7$ & $<0.01$ \\
\hline \multicolumn{4}{|l|}{ Frequency domain } \\
\hline $\mathrm{LF}\left(\mathrm{ms}^{2}\right)$ & $1.9 \pm 0.2$ & $2.5 \pm 0.2$ & 0.11 \\
\hline $\mathrm{HF}\left(\mathrm{ms}^{2}\right)$ & $6.8 \pm 1.5$ & $11.6 \pm 1.2$ & 0.03 \\
\hline LF (n.u.) & $6.1 \pm 0.9$ & $6.1 \pm 0.4$ & 1.00 \\
\hline HF (n.u.) & $19.8 \pm 3.4$ & $32.1 \pm 4.2$ & 0.04 \\
\hline LF/HF & $0.30 \pm 0.02$ & $0.23 \pm 0.02$ & 0.04 \\
\hline \multicolumn{3}{|c|}{ Heart rate variability analysis - awake } & Time domain \\
\hline SDNN (ms) & $21.0 \pm 1.4$ & $23.8 \pm 1.7$ & 0.25 \\
\hline RMSSD (ms) & $3.8 \pm 0.3$ & $5.3 \pm 0.3$ & $<0.01$ \\
\hline pNN5 (\%) & $15.3 \pm 2.9$ & $26.1 \pm 2.0$ & $<0.001$ \\
\hline \multicolumn{4}{|l|}{ Frequency domain } \\
\hline $\mathrm{LF}\left(\mathrm{ms}^{2}\right)$ & $2.2 \pm 0.2$ & $2.7 \pm 0.1$ & 0.43 \\
\hline $\mathrm{HF}\left(\mathrm{ms}^{2}\right)$ & $6.2 \pm 1.4$ & $11.3 \pm 1.3$ & $<0.01$ \\
\hline LF (n.u.) & $6.4 \pm 0.8$ & $6.6 \pm 0.4$ & 0.46 \\
\hline HF (n.u.) & $18.5 \pm 3.7$ & $31.9 \pm 4.3$ & 0.04 \\
\hline $\mathrm{LF} / \mathrm{HF}$ & $0.30 \pm 0.03$ & $0.21 \pm 0.02$ & 0.04 \\
\hline \multicolumn{3}{|c|}{ Heart rate variability analysis - asleep } & Time domain \\
\hline SDNN (ms) & $22.7 \pm 1.7$ & $26.4 \pm 1.4$ & 0.14 \\
\hline RMSSD (ms) & $4.2 \pm 0.3$ & $6.0 \pm 0.3$ & $<0.01$ \\
\hline pNN5 (\%) & $18.7 \pm 2.5$ & $31.8 \pm 2.3$ & $<0.01$ \\
\hline \multicolumn{4}{|l|}{ Frequency domain } \\
\hline $\mathrm{LF}\left(\mathrm{ms}^{2}\right)$ & $1.6 \pm 0.2$ & $2.2 \pm 0.2$ & 0.42 \\
\hline $\mathrm{HF}\left(\mathrm{ms}^{2}\right)$ & $7.4 \pm 1.6$ & $13.5 \pm 1.6$ & 0.03 \\
\hline LF (n.u.) & $5.9 \pm 1.0$ & $6.2 \pm 0.4$ & 0.79 \\
\hline HF (n.u.) & $21.2 \pm 4.2$ & $34.6 \pm 4.2$ & 0.04 \\
\hline LF/HF & $0.32 \pm 0.03$ & $0.23 \pm 0.03$ & 0.04 \\
\hline
\end{tabular}

The values are expressed as means \pm standard error of the mean; $\mathrm{p}$-values refer to between-group comparisons based on the unpaired Student's t-test.

$H F$ - high-frequency $(0.6-2.5 \mathrm{~Hz})$ signals; HR - heart rate; $L F$ - low-frequency (0.3-0.6 Hz) signals; LF/HF - the ratio of low-to high-frequency components; n.u. normalized units; pNN5 - percentage of successive RR intervals that differed by > $5 \mathrm{~ms}$; $R M S S D$ - root mean square of successive RR-interval differences; SDNN - standard deviation of normal $R R$ intervals.

rate of the sinus node to parasympathetic stimulation was significantly affected by both carbamylcholine concentration $(p=0.04)$ and ivabradine administration $(p<0.01)$, and that there was no interaction between the two factors $(p=0.93)$.

\section{DISCUSSION}

The ANS is one of the most important contributors to HR regulation, and, although several subcellular components contribute to $\mathrm{HR}$ regulation by the ANS, including the acetylcholine-dependent potassium current, calcium currents, and the $\mathrm{Na}^{+} / \mathrm{Ca}^{2+}$ exchanger, much of this regulation is achieved via $I_{\mathrm{f}}$ modulation (DiFrancesco, 2010). The ANS is, however, a double path (afferent and efferent) neural system and any significant change in cardiovascular parameters is naturally followed by adaptive changes in ANS functioning. The autonomic control of $I_{\mathrm{f}}$ and the molecular mechanisms underlying $I_{\mathrm{f}}$ modulation by the sympathetic and the parasympathetic nervous systems are well understood (DiFrancesco, 2010). Conversely, the effects of chronic $I_{\mathrm{f}}$ blockade on the ANS and on the HR response to acute autonomic changes are still largely unknown. Our study demonstrates that chronic ivabradine administration enhances vagal modulation and shifts the autonomic balance toward vagal dominance in healthy rats. Moreover, we show that chronic $I_{\mathrm{f}}$ blockade using ivabradine increases right atrial HCN4 expression, reduces the HR response to direct muscarinic receptors stimulation, cancels the cardioinhibitory response and blunts the hemodynamic response to in situ vagal stimulation. These data bring new insights into the mechanisms of ivabradine-related atrial proarrhythmia and provide evidence that long-term $I_{\mathrm{f}}$ blockade may protect against excessive bradycardia induced by acute vagal activation.

\section{Chronic Ivabradine Administration Increases Vagal Modulation and Shifts the Autonomic Balance Toward Vagal Dominance. Potential Implications for Ivabradine-Related Atrial Proarrhythmia}

Autonomic imbalance with sympathetic hyperactivity is a common denominator of a wide variety of cardiovascular diseases (Brook and Julius, 2000; Hellstrom, 2007; Floras and Ponikowski, 2015). In settings such as heart failure, sympathetic hyperactivity develops as an adaptive mechanism aiming to preserve the cardiac output (Floras and Ponikowski, 2015). However, over the long term, this increased sympathetic activity significantly contributes to impaired prognosis and increased mortality rates (Floras, 2009; Jacobson et al., 2010; Fallavollita et al., 2014). Meanwhile, strategies aiming to decrease the sympathetic and/or increase the parasympathetic tone are believed to have great potential (van Bilsen et al., 2017), and betablockers, which decrease the cardiac effects of sympathetic activation and circulating catecholamine, have already been shown to increase survival and improve prognosis in heart failure (Hjalmarson et al., 1999; Lechat et al., 1999; Flather et al., 2005).

Accumulating data suggest that "pure" HR-lowering agents could provide similar benefits (Swedberg et al., 2010) and clinical and experimental studies suggest that this beneficial effect could be at least partly due to the ability of $I_{\mathrm{f}}$ blockers to improve HRV parameters and to induce sustained increase in vagal tone (Mangin et al., 1998; Milliez et al., 2009; Kurtoglu et al., 2014; Böhm et al., 2015; El-Naggar et al., 2018), although this was not the case in the study by Silva et al. (Silva et al., 2016). However, in this latter study, ivabradine was administered intraperitoneally, for only 7-8 days, and the magnitude of $\mathrm{HR}$ reduction induced by ivabradine was significantly greater $(28 \%)$ than that achieved in the present study (13.3\%) and in the large clinical trials (15-20\%) (Swedberg et al., 2010). This increased drop in HR was also translated into a significant decrease in SBP in the ivabradine- 

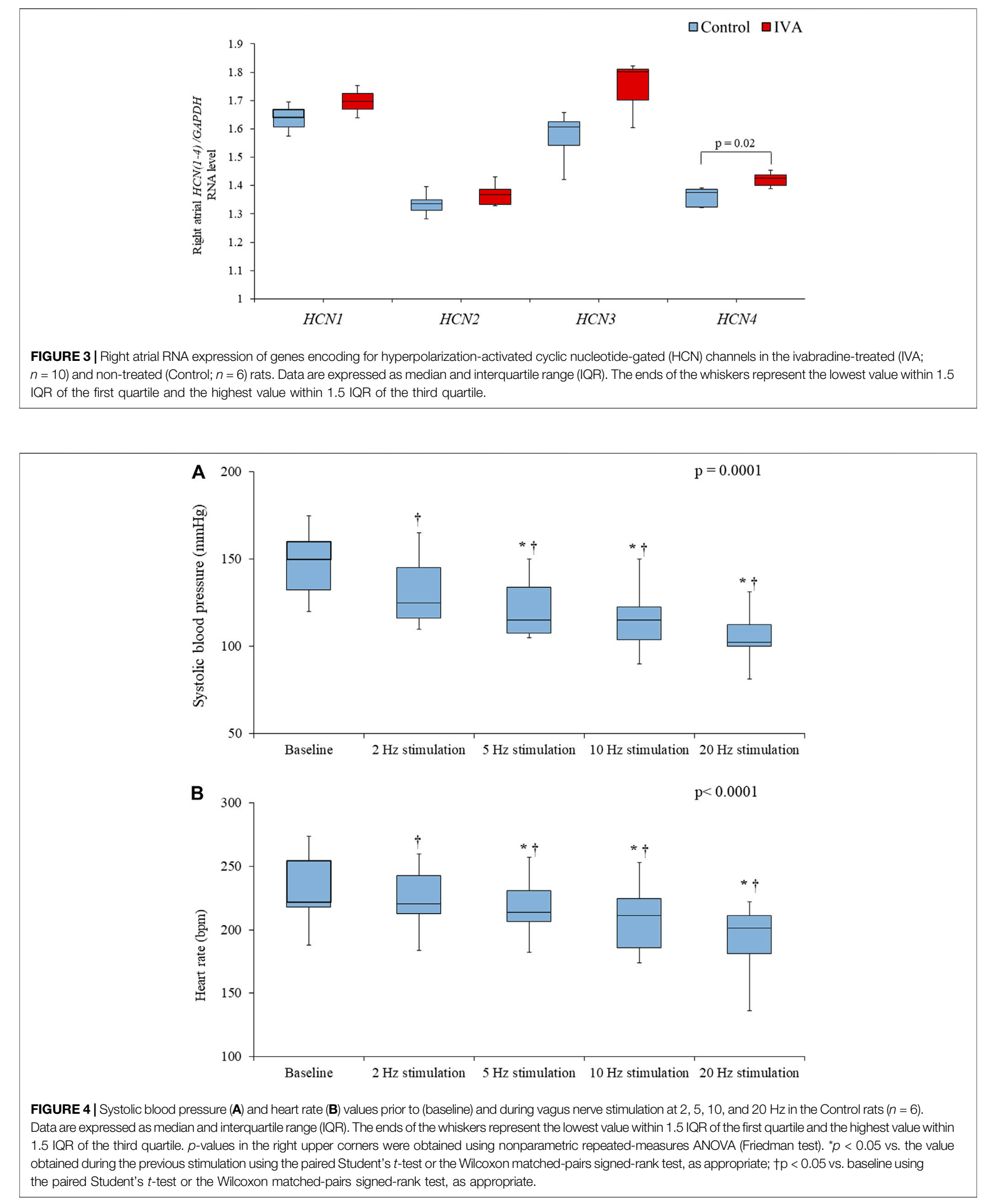

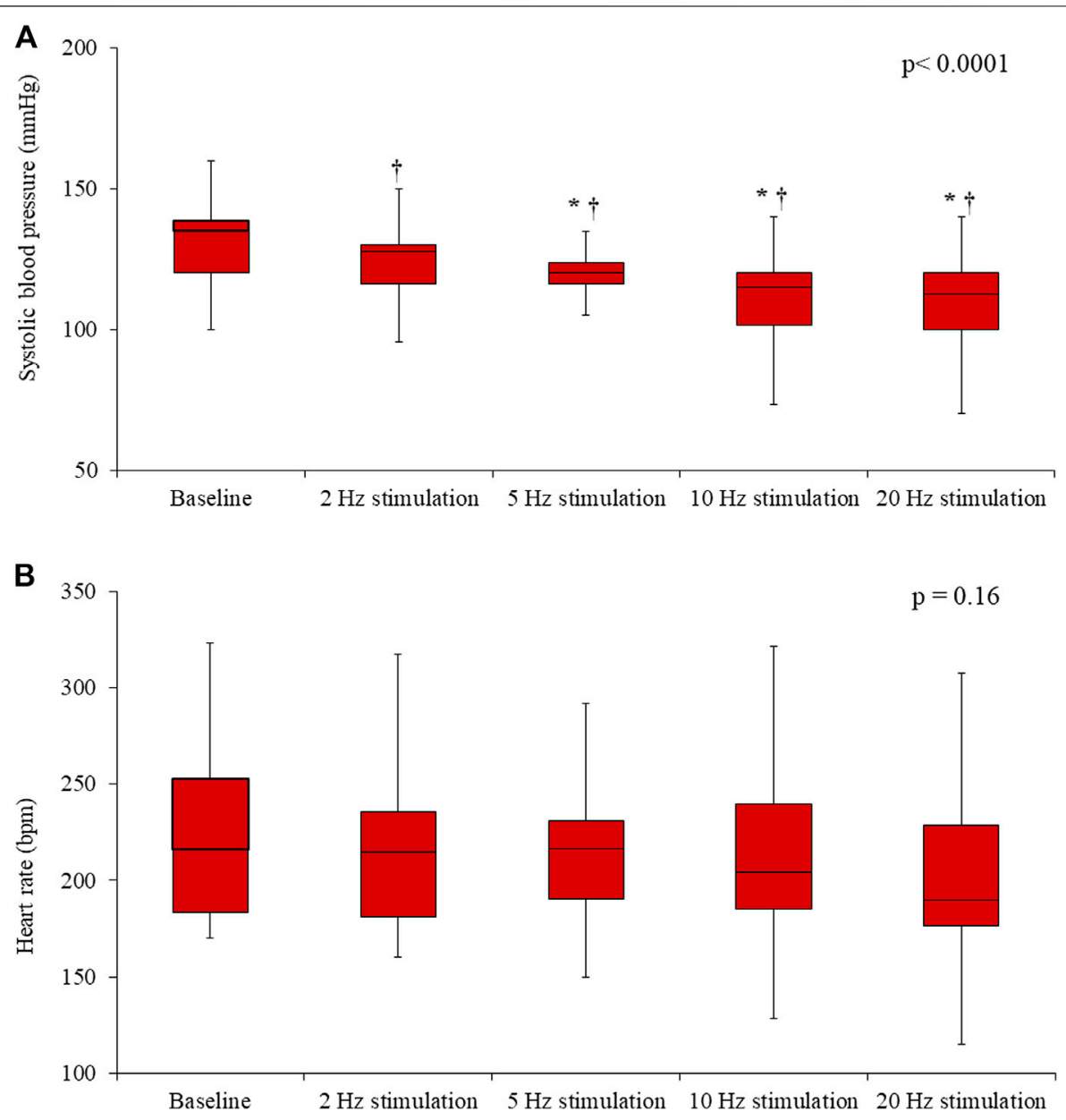

FIGURE 5 | Systolic blood pressure (A) and heart rate (B) values prior to (baseline) and during vagus nerve stimulation at 2, 5, 10, and $20 \mathrm{~Hz}$ in the ivabradinetreated rats $(n=10)$. Data are expressed as median and interquartile range (IQR). The ends of the whiskers represent the lowest value within 1.5 IQR of the first quartile and the highest value within $1.5 \mathrm{IQR}$ of the third quartile. $p$-values in the right upper corners were obtained using nonparametric repeated-measures ANOVA (Friedman test). ${ }^{*} \underline{p}<0.05$ vs. the value obtained during the previous stimulation using the paired Student's $t$-test or the Wilcoxon matched-pairs signed-rank test, as appropriate; $\dagger p<0.05$ vs. baseline using the paired Student's $t$-test or the Wilcoxon matched-pairs signed-rank test, as appropriate.

treated rats (Silva et al., 2016), effect that is not encountered at clinically relevant ivabradine doses (Swedberg et al., 2010). Although the exact mechanisms that underlie this effect remain to date unknown, the increase in vagal modulation induced by $I_{\mathrm{f}}$ blockade could be related to the lengthening of the diastolic filling time and to the consequent ventricular stretch, leading to mechanoreceptors stimulation and thus increasing the vagal and decreasing the sympathetic tone (Oren et al., 1993).

Given the well-known association between heart failure and sympathetic hyperactivity, this shift in autonomic balance toward vagal dominance was interpreted in the previous studies as an ivabradine-induced "improvement" in sympatho-vagal balance (Kurtoglu et al., 2014; Böhm et al., 2015). Although this may be true in settings associated with sympathetic hyperactivity, our data show that chronic ivabradine administration in clinically relevant doses exerts similar effects in healthy subjects. In the present study, chronic ivabradine therapy significantly reduced the HR in $24 \mathrm{~h}$, awake, and asleep recordings, without causing significant dampening of circadian variations in HR. In addition, chronic ivabradine therapy significantly increased vagal modulation (as reflected by the higher parasympathetic indexes RMSSD and pNN5, and the increased HF components of the HRV spectrum), and shifted the sympatho-vagal balance toward vagal dominance (as reflected by the significantly lower LF/HF ratio) in healthy rats. These changes were present both when the animals were awake and while asleep. This demonstrates that the increase in vagal modulation induced by long-term $I_{\mathrm{f}}$ blockade is independent on the baseline status of the autonomic balance and is not restricted to settings associated with sympathetic hyperactivity. The autonomic changes induced by ivabradine cannot be therefore interpreted as "corrective"; rather, vagal hyperactivity appears to be a common ivabradine "side effect."

\section{Clinical Implications}

On the one hand, the fact that the increase in vagal modulation induced by ivabradine is not restricted to the heart failure setting 


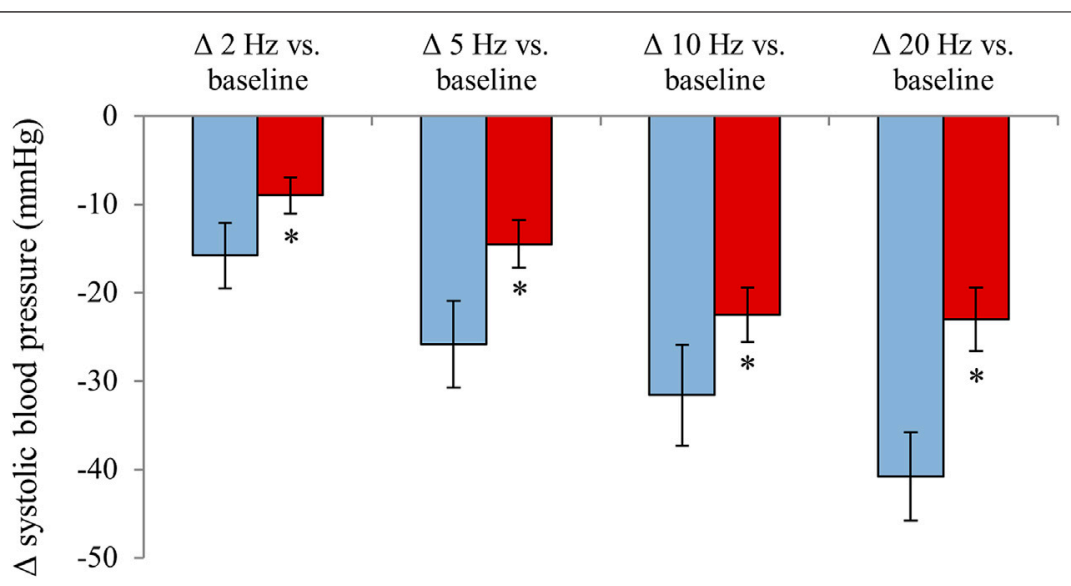

$\square$ Control $\square$ IVA

FIGURE 6 | Systolic blood pressure changes during vagus nerve stimulation at 2, 5, 10, and $20 \mathrm{~Hz}$ compared with the baseline values in the ivabradine-treated (IVA; $n=10$ ) and non-treated (Control; $n=6$ ) rats. Data are expressed as means \pm standard error of the mean. ${ }^{\star} p<0.05$ for IVA vs. Control rats using the paired Student's $t$-test or the Mann-Whitney $U$ test, as appropriate.

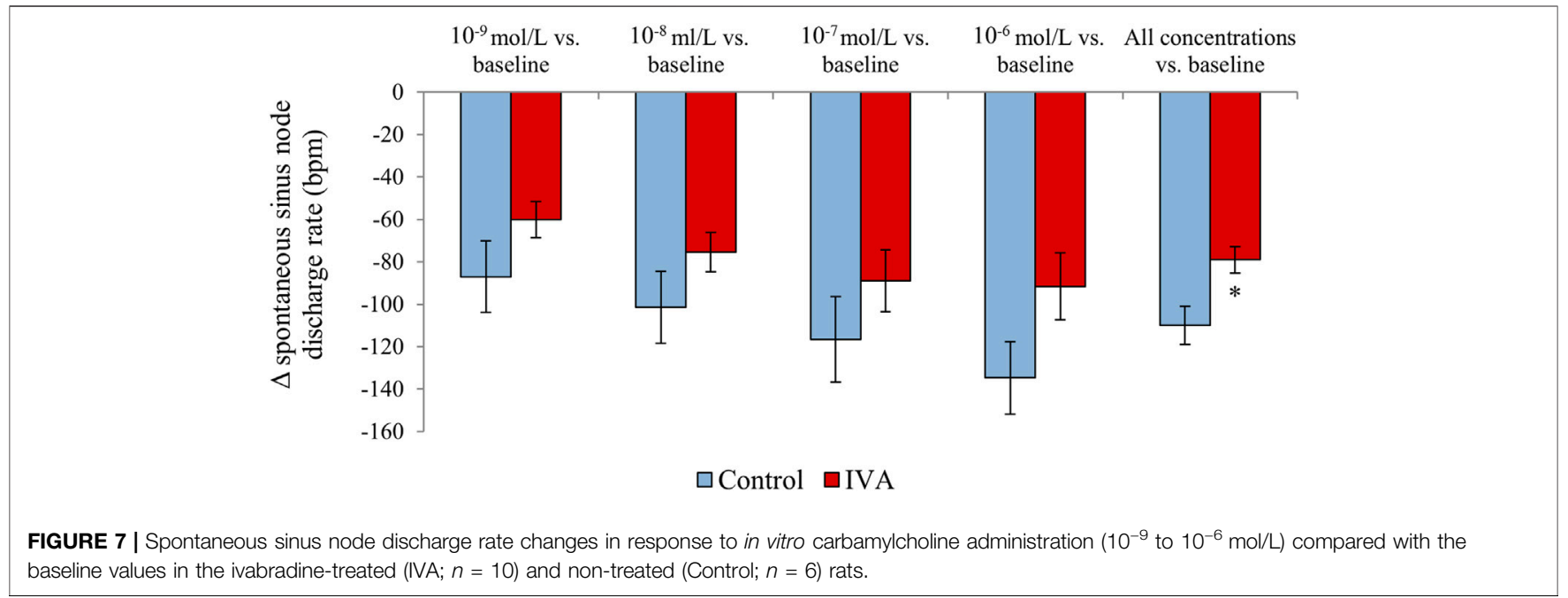

suggests that the beneficial effects of ivabradine could extend to other settings associated with increased sympathetic activity, in which the ivabradine-induced increase in vagal modulation could reduce myocardial oxygen demand and ischemia, diminish sympathetic stimulation and adrenoreceptor-mediated cytotoxicity, apoptosis, and hypertrophy, and reduce the likelihood of ventricular tachyarrhythmias and sudden death (Böhm et al., 2015). On the other hand, vagal hyperactivity has been shown to promote ectopic activity, reentry, and atrial fibrillation via multiple mechanisms (Scridon et al., 2018). Given the highly proarrhythmic effects of vagal activation at the atrial level, the increase in vagal modulation induced by ivabradine demonstrated in the present study could provide an explanation for the increased risk of atrial fibrillation associated with ivabradine use in clinical trials (Martin et al., 2014; Cammarano et al., 2016).

\section{Chronic Ivabradine Administration Cancels the Cardioinhibitory Response and Blunts the Hemodynamic Response to Acute Vagal Stimulation. Potential Implications for Vaso-Vagal Syncope}

According to estimates, $40 \%$ of individuals experience at least one episode of syncope during their lifetime (Colman et al., 2004), with vaso-vagal syncope representing up to $60 \%$ of all syncope cases (Brignole et al., 2006). Yet, the therapeutic management of vaso-vagal syncope remains highly challenging.

In the present study, in situ preganglionic vagus nerve stimulation elicited significant arterial hypotension and bradycardia in control rats. However, in the ivabradine-treated rats, vagal stimulation was not followed by the typical decrease in HR and the drop in SBP was significantly lower than that recorded in the control rats. Thus, the 
present study demonstrates for the first time that chronic ivabradine administration in clinically relevant doses cancels the cardioinhibitory response and blunts the hemodynamic response to acute vagal stimulation in rats. Interestingly, reduced mean blood pressure response and almost complete elimination of HR response to vagus nerve stimulation was also reported following metoprolol administration (Gierthmuehlen and Plachta, 2016). Meanwhile, in apparent contradiction with our data, a higher drop in HR induced by vagus nerve stimulation was reported following ivabradine administration in two recent studies (Uemura et al., 2017; Kawada et al., 2019). Although the use of different anesthetic drugs may account for some of this discrepancy, in both those studies, acute ivabradine administration was used and the drug was administered in a single, intravenous dose. Chronic and acute ivabradine administration therefore appear to exhibit discordant effects on the HR response to vagal stimulation, suggesting that chronic ivabradine therapy could induce remodeling of one or several components of the parasympathetic nervous system-cholinergic receptors- $I_{\mathrm{f}}$ axis over the long term.

Head-to-head comparisons of sinus node discharge rate responses to direct cholinergic receptors stimulation for each carbamylcholine concentration did not show significant differences between the two groups. However, when the responses to all carbamylcholine concentrations were considered together, the decrease in the spontaneous discharge rate of the sinus node was significantly lower in the ivabradine-treated compared with the control rats. Two-factor ANOVA also demonstrated that the response of the spontaneous discharge rate of the sinus node to parasympathetic stimulation was significantly affected by ivabradine administration. Thus, the cancellation of the HR response to vagal stimulation induced by ivabradine appears to result from local, receptor and/ or post-receptor ivabradine-parasympathetic nervous system interferences. In the same vein, our transcriptomic data showed that chronic ivabradine administration significantly up-regulates right atrial HCN4 expression. In previous studies, ivabradine administration counteracted the increase in ventricular $\mathrm{HCN} 4$ expression in post-myocardial infarction rats (Suffredini et al., 2012). Similarly, ivabradine has been shown to decrease HCN2 and HCN4 expression in a mixture of right and left atrial myocytes obtained from transgenic mice overexpressing the (pro) renin receptor (Wang et al., 2019). However, in the study by Leoni et al., 3 weeks ivabradine administration in mice led to a significant increase in sinus node HCN4 expression (Leoni et al., 2006). The right atrial samples examined in our study contained both nodal and nonnodal tissue. However, since HCN4 is highly expressed in the nodal pacemaker cells and only sparsely present in the remaining atrial myocardium (Chandler et al., 2009; Li et al., 2014), it is likely that the HCN4 changes observed in our study reflect sinus node, rather than non-nodal alterations. Thus, in line with the data reported by Leoni et al. (Leoni et al., 2006), our data indicate that, contrary to its effects on non-pacemaker cells, long-term ivabradine therapy augments HCN4 expression at the level of the sinus node. Eventually, HCN4 and the consequent $I_{\mathrm{f}}$ up-regulation could render the sinus node less sensitive to acute vagal inputs and protect against excessive vagalinduced bradycardia. The recent finding of Kozasa et al. that HCN4 overexpression attenuates the bradycardic response to vagus nerve stimulation (Kozasa et al., 2018) strongly supports this hypothesis.
Ivabradine does not pass the brain-blood barrier (Postea and Biel, 2011) and our data showed that long-term ivabradine therapy does not affect the expression of the neuronal specific HCN3 channel, at least at the level of the heart. However, HCN channels are also expressed in peripheral autonomic and somatosensory neurons, where they are responsible for generating the neuronal homologous of $I_{\mathrm{f}}-I_{\mathrm{h}}$, on which ivabradine has also been shown to exhibit significant inhibition (Savelieva and Camm, 2006; León-Hernández et al., 2016). An inhibiting effect of ivabradine on vagus nerve $I_{\mathrm{h}}$ activity could therefore also contribute to the lack of the HR response to in situ vagal stimulation observed in the ivabradine-treated rats.

\section{Clinical Implications}

The present study indicates for the first time that ivabradine is not only safe, but is also highly effective in preventing exaggerated vagalinduced bradycardia in rats. These data serve as a basis for future studies that will have to assess the validity of these findings in humans. If confirmed in clinical settings, this protective effect of ivabradine may place ivabradine as a drug that should not only not be avoided in patients with cardioinhibitory vaso-vagal syncope, but could even protect these patients from exaggerated vagal-induced cardioinhibitory response and reduce the risk of vaso-vagal syncope.

\section{Strengths and Limitations}

In the present study, the effects of long-term $I_{\mathrm{f}}$ blockade on autonomic function and on the cardiovascular response to parasympathetic stimulation were assessed by the use of clinically relevant ivabradine dose and route of administration. Both in situ and in vitro studies were performed, providing a comprehensive view on the impact of ivabradine therapy on the cardiovascular response to parasympathetic activation. The present study indicates ivabradineinduced sinus node HCN4 up-regulation as potential mechanism for the blunted cardiovascular response to vagal stimulation seen in these rats. However, further studies are needed to explore other potential mechanisms responsible for this effect. $H C N$ expression was not specifically assessed at the level of the sinus node; RNA levels were analyzed using a mixture of nodal and non-nodal right atrial tissue. HCN4 changes observed in our study could therefore reflect not only sinus node, but also atrial myocardial changes. However, since HCN4 is highly expressed in the nodal pacemaker cells and only sparsely present in the atrial myocardium (Chandler et al., 2009; Li et al., 2014), it is likely that the HCN4 changes reflect sinus node, rather than non-nodal HCN4 alterations. HCN changes were only assessed by RNA quantification. Due to the small size of the rat right atrium, additional tissue analyses could not be performed; changes at the protein and $I_{\mathrm{f}}$ levels were not investigated. Although altered gene expression is expected to result in protein variations, posttranscriptional and post-translational regulatory mechanisms may also influence protein level and function. Diastolic blood pressure was not assessed in the present study. Although diastolic blood pressure changes are also reflected in the SBP, a potential direct impact of ivabradine on the vagal-induced vasodepressor response cannot be excluded. However, given that ivabradine is a pure HCN channels blocker, direct interference at the vascular level is highly unlikely. In the present study, vagal modulation of the HR was evaluated using HRV analysis. Vagal tone evaluation using muscarinic receptors 
blockade, the Goldberger index, or vagal nerve activity recordings would have also been of interest. Finally, we acknowledge that by causing reduction in baseline HR, ketamine anesthesia could have diminished the impact of vagus nerve stimulation on the HR. However, whereas ketamine anesthesia manifested similar effects on the HR in the ivabradine-treated and non-treated rats, the HR response to vagus nerve stimulation was only abolished in the IVA rats. Thus, the lack of $\mathrm{HR}$ response to vagus nerve stimulation observed in the ivabradine-treated rats cannot be ascribed to the effects of ketamine anesthesia.

\section{CONCLUSION}

The present study demonstrates that long-term ivabradine therapy produces a significant increase in vagal modulation and shifts the sympatho-vagal balance toward vagal dominance. This increase in vagal modulation induced by ivabradine could contribute to the improved outcomes observed in patients with sympathetic hyperactivity, but could also provide an explanation for the increased risk of atrial fibrillation associated with ivabradine therapy in clinical trials. Ivabradine abolished the cardioinhibitory and blunted the hemodynamic response to acute vagal activation in rats, suggesting that $I_{\mathrm{f}}$ blockade may emerge as a promising therapy for patients with cardioinhibitory vaso-vagal syncope. Our data indicate sinus node $\mathrm{HCN} 4$ up-regulation as a potential mechanism underlying the protective effect of ivabradine against excessive vagalinduced bradycardia in rats.

\section{REFERENCES}

Böhm, M., Borer, J. S., Camm, J., Ford, I., Lloyd, S. M., Komajda, M., et al. (2015). Twenty-four-hour heart rate lowering with ivabradine in chronic heart failure: insights from the SHIFT Holter substudy. Eur. J. Heart Fail. 17 (5), 518-526. doi:10.1002/ejhf.258

Brignole, M., Menozzi, C., Bartoletti, A., Giada, F., Lagi, A., Ungar, A., et al. (2006). A new management of syncope: prospective systematic guideline-based evaluation of patients referred urgently to general hospitals. Eur. Heart J. 27 (1), 76-82. doi:10.1093/eurheartj/ehi647

Brook, R. D., and Julius, S. (2000). Autonomic imbalance, hypertension, and cardiovascular risk. Am. J. Hypertens. 13 (6 Pt 2), 112S-122S. doi:10.1016/ s0895-7061(00)00228-4

Camm, A. J., and Lau, C.-P. (2003). Electrophysiological effects of a single intravenous administration of ivabradine (S 16257) in adult patients with normal baseline electrophysiology. Drugs R. andD 4 (2), 83-89. doi:10.2165/ 00126839-200304020-00001

Cammarano, C., Silva, M., Comee, M., Donovan, J. L., and Malloy, M. J. (2016). Meta-analysis of ivabradine in patients with stable coronary artery disease with and without left ventricular dysfunction. Clin. Ther. 38, 387-395. doi:10.1016/j. clinthera.2015.12.018

Cappato, R., Castelvecchio, S., Ricci, C., Bianco, E., Vitali-Serdoz, L., GnecchiRuscone, T., et al. (2012). Clinical efficacy of ivabradine in patients with inappropriate sinus tachycardia. J. Am. Coll. Cardiol. 60 (15), 1323-1329. doi:10.1016/j.jacc.2012.06.031

Chandler, N. J., Greener, I. D., Tellez, J. O., Inada, S., Musa, H., Molenaar, P., et al. (2009). Molecular architecture of the human sinus node. Circulation 119 (12), 1562-1575. doi:10.1161/CIRCULATIONAHA.108.804369

Colman, N., Nahm, K., Ganzeboom, K. S., Shen, W. K., Reitsma, J., Linzer, M., et al. (2004). Epidemiology of reflex syncope. Clin. Auton. Res. 14 (Suppl. 1), i9-i17. doi:10.1007/s10286-004-1003-3

\section{DATA AVAILABILITY STATEMENT}

The raw data supporting the conclusion of this article will be made available by the authors, without undue reservation.

\section{ETHICS STATEMENT}

The animal study was reviewed and approved by the local Ethics Committee and the National Sanitary Veterinary and Food Safety Authority.

\section{AUTHOR CONTRIBUTIONS}

AS and RCS contributed to conception and design of the work. AS drafted the manuscript. AS, VBH, AIB, DAC, VM, CB, MP, and RCS contributed to acquisition, analysis or interpretation of data for the work. VBH, AIB, DAC, VM, CB, MP, and RCS performed critical revision of the manuscript for important intellectual content. All authors approved the final version of the manuscript.

\section{FUNDING}

This work was supported by the University of Medicine, Pharmacy, Science and Technology "George Emil Palade" of Târgu Mureș Research Grant number 615/2/17.01.2019.

DiFrancesco, D. (2010). The role of the funny current in pacemaker activity. Circ. Res. 106 (3), 434-446. doi:10.1161/CIRCRESAHA.109.208041

Doñate Puertas, R., Meugnier, E., Romestaing, C., Rey, C., Morel, E., Lachuer, J., et al. (2017). Atrial fibrillation is associated with hypermethylation in human left atrium, and treatment with decitabine reduces atrial tachyarrhythmias in spontaneously hypertensive rats. Translational Res. 184, 57-67. doi:10.1016/j. trsl.2017.03.004e5

El-Naggar, A. E., El-Gowilly, S. M., and Sharabi, F. M. (2018). Possible ameliorative effect of ivabradine on the autonomic and left ventricular dysfunction induced by doxorubicin in male rats. J. Cardiovasc. Pharmacol. 72 (1), 22-31. doi:10. 1097/FJC.0000000000000586

Fallavollita, J. A., Heavey, B. M., Luisi, A. J., Jr, Michalek, S. M., Baldwa, S., Mashtare, T. L., Jr, et al. (2014). Regional myocardial sympathetic denervation predicts the risk of sudden cardiac arrest in ischemic cardiomyopathy. J. Am. Coll. Cardiol. 63 (2), 141-149. doi:10.1016/j.jacc.2013.07.096

Flather, M. D., Shibata, M. C., Coats, A. J. S., Van Veldhuisen, D. J., Parkhomenko, A., Borbola, J., et al. (2005). Randomized trial to determine the effect of nebivolol on mortality and cardiovascular hospital admission in elderly patients with heart failure (SENIORS). Eur. Heart J. 26 (3), 215-225. doi:10. 1093/eurheartj/ehi115

Floras, J. S., and Ponikowski, P. (2015). The sympathetic/parasympathetic imbalance in heart failure with reduced ejection fraction. Eur. Heart J. 36 (30), 1974-1982. doi:10.1093/eurheartj/ehv087

Floras, J. S. (2009). Sympathetic nervous system Activation in human heart failure. J. Am. Coll. Cardiol. 54 (5), 375-385. doi:10.1016/j.jacc.2009.03.061

Gallet, C., Chapuis, B., Oréa, V., Scridon, A., Barrès, C., Chevalier, P., et al. (2013). Automatic atrial arrhythmia detection based on RR interval analysis in conscious rats. Cardiovasc. Eng. Tech. 4 (4), 535-543. doi:10.1007/s13239013-0150-X

Gee, M. E., Watkins, A. K., Brown, J. N., and Young, E. J. A. (2018). Ivabradine for the treatment of postural orthostatic tachycardia syndrome: a systematic review. Am. J. Cardiovasc. Drugs 18 (3), 195-204. doi:10.1007/s40256-017-0252-1 
Gierthmuehlen, M., and Plachta, D. T. T. (2016). Effect of selective vagal nerve stimulation on blood pressure, heart rate and respiratory rate in rats under metoprolol medication. Hypertens. Res. 39 (2), 79-87. doi:10.1038/hr.2015.122

Hellstrom, H. R. (2007). The altered homeostatic theory: a hypothesis proposed to be useful in understanding and preventing ischemic heart disease, hypertension, and diabetes-including reducing the risk of age and atherosclerosis. Med. Hypotheses 68 (2), 415-433. doi:10.1016/j.mehy.2006.05.031

Hjalmarson, A., Goldstein, S., Fagerberg, B., Wedel, H., Waagstein, F., Kjekshus, J., et al. (1999). Effect of metoprolol CR/XL in chronic heart failure: metoprolol $\mathrm{CR} / \mathrm{XL}$ randomised intervention trial in congestive heart failure (MERIT-HF). Lancet 353 (9169), 2001-2007.

Jacobson, A. F., Senior, R., Cerqueira, M. D., Wong, N. D., Thomas, G. S., Lopez, V. A., et al. (2010). Myocardial iodine-123 meta-iodobenzylguanidine imaging and cardiac events in heart failure. J. Am. Coll. Cardiol. 55 (20), 2212-2221. doi:10. 1016/j.jacc.2010.01.014

Kawada, T., Yamamoto, H., Uemura, K., Hayama, Y., Nishikawa, T., and Sugimachi, M. (2019). Intravenous ivabradine augments the dynamic heart rate response to moderate vagal nerve stimulation in anesthetized rats. Am. J. Physiology-Heart Circulatory Physiol. 317 (3), H597-H606. doi:10.1152/ ajpheart.00288.2019

Kozasa, Y., Nakashima, N., Ito, M., Ishikawa, T., Kimoto, H., Ushijima, K., et al. (2018). HCN4 pacemaker channels attenuate the parasympathetic response and stabilize the spontaneous firing of the sinoatrial node. J. Physiol. 596 (5), 809-825. doi:10.1113/JP275303

Kurtoglu, E., Balta, S., Karakus, Y., Yasar, E., Cuglan, B., Kaplan, O., et al. (2014). Ivabradine improves heart rate variability in patients with nonischemic dilated cardiomyopathy. Arq Bras Cardiol. 103, 308-314. doi:10.5935/abc.20140109

Lechat, P., Brunhuber, K. W., Hofmann, R., Kuhn, P., Nesser, H. J., Slany, J., et al. (1999). The cardiac insufficiency bisoprolol study II (CIBIS-II): a randomised trial. Lancet 353 (9146), 9-13.

León-Hernández, J. V., Martín-Pintado-Zugasti, A., Frutos, L. G., Alguacil-Diego, I. M., de la Llave-Rincón, A. I., and Fernandez-Carnero, J. (2016). Immediate and short-term effects of the combination of dry needling and percutaneous TENS on post-needling soreness in patients with chronic myofascial neck pain. Braz. J. Phys. Ther. 20 (5), 422-431. doi:10.1590/bjpt-rbf.2014.0176

Leoni, A.-L., Marionneau, C., Demolombe, S., Bouter, S. L., Mangoni, M. E., Escande, D., et al. (2006). Chronic heart rate reduction remodels ion channel transcripts in the mouse sinoatrial node but not in the ventricle. Physiol. Genomics 24 (1), 4-12. doi:10.1152/physiolgenomics.00161.2005

Li, Y. D., Hong, Y. F., Zhang, Y., Zhou, X. H., Ji, Y. T., Li, H. L., et al. (2014). Association between reversal in the expression of hyperpolarization-activated cyclic nucleotide-gated (HCN) channel and age-related atrial fibrillation. Med. Sci. Monit. 20, 2292-2297. doi:10.12659/MSM.892505

Mangin, L., Swynghedauw, B., Benis, A., Thibault, N., Lerebours, G., and Carré, F. (1998). Relationships between heart rate and heart rate variability: study in conscious rats. J. Cardiovasc. Pharmacol. 32 (4), 601-607. doi:10.1097/ 00005344-199810000-00012

Manz, M., Reuter, M., Lauck, G., Omran, H., and Jung, W. (2003). A single intravenous dose of ivabradine, a novel if inhibitor, lowers heart rate but does not depress left ventricular function in patients with left ventricular dysfunction. Cardiology 100 (3), 149-155. doi:10.1159/000073933

Martin, R. I. R., Pogoryelova, O., Koref, M. S., Bourke, J. P., Teare, M. D., and Keavney, B. D. (2014). Atrial fibrillation associated with ivabradine treatment: meta-analysis of randomised controlled trials. Heart 100, 1506-1510. doi:10. 1136/heartjnl-2014-305482

Milliez, P., Messaoudi, S., Nehme, J., Rodriguez, C., Samuel, J.-L., and Delcayre, C. (2009). Beneficial effects of delayed ivabradine treatment on cardiac anatomical and electrical remodeling in rat severe chronic heart failure. Am. J. PhysiologyHeart Circulatory Physiol. 296, H435-H441. doi:10.1152/ajpheart.00591.2008

Oren, R. M., Schobel, H. P., Weiss, R. M., Stanford, W., and Ferguson, D. W. (1993). Importance of left atrial baroreceptors in the cardiopulmonary baroreflex of normal humans. J. Appl. Physiol. 74 (6), 2672-2680. doi:10. 1152/jappl.1993.74.6.26721993

Postea, O., and Biel, M. (2011). Exploring HCN channels as novel drug targets. Nat. Rev. Drug Discov. 10 (12), 903-914. doi:10.1038/nrd3576
Sartiani, L., Mannaioni, G., Masi, A., Novella Romanelli, M., and Cerbai, E. (2017). The hyperpolarization-activated cyclic nucleotide-gated channels: from biophysics to pharmacology of a unique family of ion channels. Pharmacol. Rev. 69 (4), 354-395. doi:10.1124/pr.117.014035

Savelieva, I., and Camm, A. J. (2006). Novel if current inhibitor ivabradine: safety considerations. Adv. Cardiol. 43, 79-96. doi:10.1159/000095430

Scridon, A., Gallet, C., Arisha, M. M., Oréa, V., Chapuis, B., Li, N., et al. (2012). Unprovoked atrial tachyarrhythmias in aging spontaneously hypertensive rats: the role of the autonomic nervous system. Am. J. Physiology-Heart Circulatory Physiol. 303 (3), H386-H392. doi:10.1152/ajpheart.00004.2012

Scridon, A., Perian, M., Marginean, A., Fisca, C., Vantu, A., Ghertescu, D., et al. (2015). Wistar rats with long-term streptozotocin-induced type 1 diabetes mellitus replicate the most relevant clinical, biochemical, and hematologic features of human diabetes/Sobolanii Wistar cu diabet zaharat tip 1 indus cu streptozotocina reproduc cele mai relevante caracteristici clinice, biochimice si hematologice ale diabetului uman. Rev. Romana Med. Lab. 23 (3), 263-274. doi:10.1515/rrlm-2015-0028

Scridon, A., Şerban, R. C., and Chevalier, P. (2018). Atrial fibrillation: neurogenic or myogenic?. Arch. Cardiovasc. Dis. 111 (1), 59-69. doi:10.1016/j.acvd.2017. 11.001

Silva, F. C., Paiva, F. A., Müller-Ribeiro, F. C., Caldeira, H. M. A., Fontes, M. A. P., de Menezes, R. C. A., et al. (2016). Chronic treatment with ivabradine does not affect cardiovascular autonomic control in rats. Front. Physiol. 7, 305. doi:10. 3389/fphys.2016.00305

Suffredini, S., Stillitano, F., Comini, L., Bouly, M., Brogioni, S., Ceconi, C., et al. (2012). Long-term treatment with ivabradine in post-myocardial infarcted rats counteracts f-channel overexpression. Br. J. Pharmacol. 165 (5), 1457-1466. doi:10.1111/j.1476-5381.2011.01627.x

Sutton, R., Salukhe, T. V., Franzen-McManus, A.-C., Collins, A., Lim, P. B., and Francis, D. P. (2014). Ivabradine in treatment of sinus tachycardia mediated vasovagal syncope. Europace 16 (2), 284-288. doi:10.1093/ europace/eut 226

Swedberg, K., Komajda, M., Böhm, M., Borer, J. S., Ford, I., Dubost-Brama, A., et al. (2010). Ivabradine and outcomes in chronic heart failure (SHIFT): a randomised placebo-controlled study. The Lancet 376 (9744), 875-885. doi:10.1016/S0140-6736(10)61198-1

Taub, P. R., Zadourian, A., Lo, H. C., Ormiston, C. K., Golshan, S., and Hsu, J. C. (2021). Randomized trial of ivabradine in patients with hyperadrenergic postural orthostatic tachycardia syndrome. J. Am. Coll. Cardiol. 77 (7), 861-871. doi:10.1016/j.jacc.2020.12.029

Uemura, K., Inagaki, M., Zheng, C., Kawada, T., Li, M., Fukumitsu, M., et al. (2017). Acute ivabradine treatment reduces heart rate without increasing atrial fibrillation inducibility irrespective of underlying vagal activity in dogs. Heart Vessels 32 (4), 484-494. doi:10.1007/s00380-016-0922-y

van Bilsen, M., Patel, H. C., Bauersachs, J., Böhm, M., Borggrefe, M., Brutsaert, D., et al. (2017). The autonomic nervous system as a therapeutic target in heart failure: a scientific position statement from the Translational Research Committee of the Heart Failure Association of the European Society of Cardiology. Eur. J. Heart Fail. 19 (11), 1361-1378. doi:10.1002/ejhf.921

Wang, J., Yang, Y. m., Li, Y., Zhu, J., Lian, H., Shao, X. h., et al. (2019). Long-term treatment with ivabradine in transgenic atrial fibrillation mice counteracts hyperpolarization-activated cyclic nucleotide gated channel overexpression. J. Cardiovasc. Electrophysiol. 30 (2), 242-252. doi:10.1111/jce.13772

Conflict of Interest: The authors declare that the research was conducted in the absence of any commercial or financial relationships that could be construed as a potential conflict of interest.

Copyright (๑) 2021 Scridon, Halațiu, Balan, Cozac, Moldovan, Bănescu, Perian and Serban. This is an open-access article distributed under the terms of the Creative Commons Attribution License (CC BY). The use, distribution or reproduction in other forums is permitted, provided the original author(s) and the copyright owner(s) are credited and that the original publication in this journal is cited, in accordance with accepted academic practice. No use, distribution or reproduction is permitted which does not comply with these terms. 